\title{
Maternal age at menarche and atopy among offspring at the age of 31 years
}

\author{
Baizhuang Xu, Marjo-Riitta Järvelin, Anna-Liisa Hartikainen, Juha Pekkanen
}

Unit of Environmental

Epidemiology,

National Public Health

Institute, 70701

Kuopio, Finland

B Xu

J Pekkanen

Department of Public Health and General Practice, University of Oulu and Department of Epidemiology and Public Health, Imperial College School of Medicine, London W2 1PG, UK M-R Järvelin

Department of Obstetrics and Gynaecology, University Hospital, University of Oulu, 90220 Oulu, Finland A-L Hartikainen

Correspondence to: Dr B Xu, Department of Epidemiology and Public Health, Imperial College School of Medicine, London W2 1PG, UK

email: b.xu@ic.ac.uk

Received 24 November 1999 Returned to authors

31 January 2000

Revised version received

31 March 2000

Accepted for publication

17 April 2000

\begin{abstract}
Background-Influences of female hormones on the occurrence of allergic disorders have been suggested. Age at menarche may be a marker of endogenous oestrogen levels. Data from a Finnish birth cohort followed to adulthood were analysed to determine whether there is any association between maternal age at menarche and the occurrence of atopy among offspring.
\end{abstract}

Methods-The study was conducted in 5188 subjects born in northern Finland for whom data collections were started during pregnancy and a follow up examination was completed at the age of 31 years. Atopy was determined by skin prick tests with cat, birch, grass, and mite extracts, and doctor diagnosed asthma was ascertained by questionnaire at follow up. Maternal age at menarche was obtained from perinatal data. Logistic regression models were used to adjust for maternal age, parity, smoking, season of birth, parental allergy, and measures of adiposity and socioeconomic status.

Results-The prevalence of atopy at the age of 31 years was lower in children whose mothers reached menarche at a later age, especially after age 15. Compared with children whose mothers started menarche at the age of 16 or over, the adjusted odds ratios of being atopic for children whose mothers started menarche younger than or at $12,13,14$ and 15 years were 1.43 (95\% CI 1.12 to 1.83$), 1.29$ (95\% CI 1.03 to 1.60$), 1.15$ (95\% CI 0.93 to 1.42$)$, and $1.19(95 \%$ CI 0.95 to 1.48$)$, respectively. Among girls, the offspring's own age at menarche was not significantly associated with atopy.

Conclusion-Our results encourage further evaluation of the potential effect of maternal age at menarche on the later development of atopy and possible biological mechanisms.

(Thorax 2000;55:691-693)

Keywords: atopy; asthma; maternal age at menarche

The prevalence of allergies is increasing in many countries, but most of the increase cannot be explained by factors so far known or identified. ${ }^{1}$ The potential influence of oestrogen on the occurrence of allergic disorders has been suggested. ${ }^{2}$ Age at menarche reflects endogenous oestrogen status. ${ }^{3}$ Early age at menarche has been shown to be associated with higher oestrogen levels among girls around the onset of puberty ${ }^{3}$ and also in adulthood. ${ }^{4}$ As a critical period in the later development of allergic disorders appears to be the prenatal and perinatal environment, we have examined the association between age at menarche of the mother and the occurrence of atopy among her offspring.

\section{Methods}

The study population was based on a geographically defined cohort of 12058 live births in northern Finland in $1966^{5}$ of whom 11635 had survived up to 31 years of age in 1997. The 8463 subjects who were still living in northern Finland or had moved to the Helsinki area (in southern Finland) were invited to a clinical examination in 1997, and 6025 of these attended. ${ }^{6}$ Sensitivity to the three most common allergens in Finland-cat, birch, and timothy grass - and also to house dust mite (Dermatophagoides pteronyssinus) was assessed by skin prick tests, together with histamine dihydrochloride $(10 \mathrm{mg} / \mathrm{ml})$ and diluent of the allergen extracts used as positive and negative controls. Skin reactions to the allergens were recorded after 15 minutes, taking the average of the maximum weal diameter and the diameter perpendicular to the maximum. Subjects with a weal reaction of $3 \mathrm{~mm}$ or greater to at least one of the four allergens tested were considered to be atopic. Twelve subjects with a positive reaction to the negative control were excluded. All subjects had a positive reaction to histamine. Information on doctor diagnosed asthma was obtained from a self-administered questionnaire that was completed by the subjects who attended for clinical examination. The final analyses were limited to 5188 subjects with information on atopy, asthma, and perinatal data and with signed consent to use their data for research.

The information on maternal age at menarche, weight, and height before pregnancy was collected from the mothers' first antenatal visit and size at birth was taken from hospital records. ${ }^{5}$ Current weight and height at 31 years were measured during the follow up clinical examinations. Ponderal index at birth was 
Table 1 Associations between maternal age at menarche and occurrence of atopy in children at 31 years

\begin{tabular}{|c|c|c|c|c|}
\hline $\begin{array}{l}\text { Maternal age at } \\
\text { menarche } \\
\text { (years) }\end{array}$ & $\begin{array}{l}\text { Total no. } \\
\text { (atopics) }\end{array}$ & $\%$ & Crude OR $(95 \%$ CI) & Adjusted $\mathrm{OR}^{\star}(95 \% \mathrm{CI})$ \\
\hline \multicolumn{5}{|l|}{ Total } \\
\hline$\leqslant 12$ & $694(242)$ & 34.9 & 1.49 (1.19 to 1.87$)$ & $1.43(1.12$ to 1.83$)$ \\
\hline 13 & $1181(387)$ & 32.8 & $1.36(1.11$ to 1.66$)$ & $1.29(1.03$ to 1.60$)$ \\
\hline 14 & $1406(424)$ & 30.2 & $1.21(0.99$ to 1.47$)$ & $1.15(0.93$ to 1.42$)$ \\
\hline 15 & $1145(344)$ & 30.0 & $1.20(0.98$ to 1.47$)$ & $1.19(0.95$ to 1.48$)$ \\
\hline$\geqslant 16$ & $762(201)$ & 26.4 & 1.00 & 1.00 \\
\hline $\mathrm{p}$ for trend & & & 0.000 & 0.005 \\
\hline \multicolumn{5}{|l|}{ Men } \\
\hline$\leqslant 12$ & 347 (133) & 38.3 & $1.44(1.06$ to 1.96$)$ & $1.32(0.94$ to 1.86$)$ \\
\hline 13 & $593(215)$ & 36.3 & $1.32(1.00$ to 1.74$)$ & $1.19(0.88$ to 1.62$)$ \\
\hline 14 & $710(231)$ & 32.5 & $1.12(0.86$ to 1.47$)$ & $1.08(0.81$ to 1.45$)$ \\
\hline 15 & $566(171)$ & 30.2 & $1.01(0.76$ to 1.33$)$ & $0.96(0.71$ to 1.31$)$ \\
\hline$\geqslant 16$ & $382(115)$ & 30.1 & 1.00 & 1.00 \\
\hline $\mathrm{p}$ for trend & & & 0.002 & 0.048 \\
\hline \multicolumn{5}{|l|}{ Women } \\
\hline$\leqslant 12$ & 347 (109) & 31.4 & $1.57(1.13$ to 2.18$)$ & $1.58(1.10$ to 2.26$)$ \\
\hline 13 & $588(172)$ & 29.3 & $1.41(1.05$ to 1.91$)$ & $1.40(1.01$ to 1.93$)$ \\
\hline 14 & 696 (193) & 27.7 & $1.31(0.98$ to 1.76$)$ & $1.24(0.90$ to 1.70$)$ \\
\hline 15 & $579(173)$ & 29.9 & 1.46 (1.08 to 1.96$)$ & 1.47 (1.07 to 2.02$)$ \\
\hline$\geqslant 16$ & $380(86)$ & 22.6 & 1.00 & 1.00 \\
\hline $\mathrm{p}$ for trend & & & 0.034 & 0.149 \\
\hline
\end{tabular}

*Adjusted variables were defined in the following ways: maternal age $\leqslant 20,21-25,26-30,31-35$, and $\geqslant 36$ years; maternal social classes I + II (professionals with the highest education and other white collar workers), III (skilled workers), IV (unskilled workers) and farmers; maternal smoking in pregnancy yes or no; parity $0,1,2-3$ and $\geqslant 4$; seasons at birth March-May, June-August, September-November and December-February; parental allergy yes if either the father or mother had allergic disorders, otherwise no; current vocational training in five categories; maternal BMI, ponderal index and current $\mathrm{BMI}$ in quintiles.

calculated using the standard formula of weight $(100 \mathrm{~g})$ divided by cube of length $\left(\mathrm{cm}^{3}\right)$, and body mass index (BMI) as weight $(\mathrm{kg})$ divided by the height squared $\left(\mathrm{m}^{2}\right)$. The information on maternal age, paternal social class, maternal smoking during pregnancy, parity, and season of birth were obtained from perinatal data, and daughter's age at menarche, current physical activity, and vocational training at the age of 31 years from a postal questionnaire.

Logistic regression models were employed to estimate the effect of maternal age at menarche on risk of atopy at the age of 31 years. In multiple analyses those variables previously reported to be associated with atopy or asthma were adjusted. The adjusted variables were defined in the following ways: maternal age as $\leqslant 20,21-25,26-30,31-35$, and $\geqslant 36$ years; paternal social classes I + II (professionals with the highest education and other white collar workers), III (skilled workers), IV (unskilled workers) and farmers; maternal smoking in pregnancy yes or no; parity $0,1,2-3$, and $\geqslant 4$; seasons at birth March-May, June-August, September-November, and DecemberFebruary; parental allergy yes if either parent had allergic disorders, otherwise no; current vocational training in five categories; maternal BMI before pregnancy, ponderal index at birth, and current $\mathrm{BMI}$ in quintiles.

The mean maternal age at menarche for the 5188 analysed subjects and for the other 3275 subjects who were invited for clinical examination but did not attend or who attended but were without complete data was 14.07 and 14.04 , respectively $(\mathrm{p}=0.34)$.

\section{Results}

The prevalence of atopy at the age of 31 years for men and women was $33 \%$ and $28 \%$, respectively. Atopy was less common in the children of mothers who had experienced menarche at a later age (table 1). After adjusting for potential confounders, the association was reduced for men but remained unchanged in women (table 1). In women the effect was seen mainly in mothers with menarche at the age of 16 or over, whereas in men a significant trend was observed in both univariate and adjusted analyses. We did not find any association between maternal age at menarche and asthma. The prevalence of doctor diagnosed asthma for maternal ages at menarche of $\leqslant 12,13,14,15$, and $\geqslant 16$ was $7.9 \%, 7.4 \%$, $7.6 \%, 6.3 \%$ and $7.7 \%$, respectively $(\mathrm{p}=0.89$, Pearson $\chi^{2}$ test) among men and $11.0 \%, 8.2 \%$, $6.5 \%, 9.3 \%$, and $8.5 \%$, respectively among women $(\mathrm{p}=0.15)$.

Maternal age at menarche was correlated with that of the daughter $(r=0.19, \mathrm{p}=0.01)$. The mean age at menarche was 14.1 years for mothers and 12.9 years for daughters $(\mathrm{p}=$ $0.00)$. The prevalence of atopy was $29.8 \%$, $27.5 \%, 27.4 \%, 26.4 \%$, and $24 \%$, respectively, for daughters with menarche at the age of $\leqslant 12$, $13,14,15$, and $\geqslant 16$ years $\left(p=0.63\right.$, Pearson $\chi^{2}$ test; $p=0.14$, test for trend). The corresponding prevalence of doctor diagnosed asthma was $8.6 \%, 9.0 \%, 7.3 \%, 8.2 \%, 12.2 \%$, respectively ( $\mathrm{p}=0.67$, Pearson $\chi^{2}$ test). After including daughter's age at menarche into the multivariate model, the association of maternal age at menarche with atopy remained unchanged.

\section{Discussion}

The results of this study suggest that children are less likely to have atopic sensitisation at the age of 31 years if their mothers reached menarche at a later age. The observed association is not strong, but seems not to be explained by adjustment for potential confounders. To our knowledge, this is the first report of such an association. However, the biological mechanism underlying this finding is not clear. Age at menarche is mainly a marker of oestrogen status, and oestrogens have been shown to enhance histamine release in rats, probably via an IgE mediated mechanism. ${ }^{7}$ Clinical observations also indicate that a skin prick test is more sensitive to histamine in both atopic and non-atopic women on days 12-16 of the menstrual cycle, corresponding to ovulation and peak oestrogen levels. ${ }^{8}$ Female sex hormones can also affect immune function through their influence on $\mathrm{T}$ cell populations, the production of specific antibodies, and proinflammatory mediators. ${ }^{9}$ It is possible that differences in the maternal oestrogen environment, represented by varying age at menarche, could programme the immune system of the fetus in a manner that could affect the atopic status later in life.

Age at menarche is one of the few established risk factors for breast cancer. Anthropometric, socioeconomic, lifestyle, and genetic factors are commonly documented as predictors for age at menarche. ${ }^{10}$ In the current analyses, maternal BMI before pregnancy, social class, and smoking during pregnancy were considered but none appeared to confound the observed association. 
Age at menarche of the mother and the daughter were significantly correlated, even though the mean age at menarche of mothers was later than for their daughters (14.1 versus 12.9 years). Daughter's age at menarche was not significantly associated with her atopic status, but there was a suggestion of a similar trend as with maternal age at menarche. This result suggests that the intrauterine environment might be more important in later life in terms of development of adult atopy, at least for the mechanism underlying the association observed here.

Of the original birth cohort born in 1966 in northern Finland, only those still living in the same area or those who had moved to the Helsinki area were invited to participate in the study; $71 \%$ attended and complete data were available for $61 \%$. However, only $45 \%$ of the original cohort was included in the present analyses. This introduces possibilities for selection bias if migration away from northern Finland or attendance for the clinical examination were associated with atopy and age at menarche. However, we consider that the possible effect of selection bias on the current analyses was only limited as we included the main area of migration (around Helsinki) and because there was no substantial difference in maternal age at menarche between those included in the analyses and other subjects (14.07 versus 14.04 years, $\mathrm{p}=0.34$ ).
In conclusion, atopy at the age of 31 years seems to be less common among those whose mothers experienced a late menarche. Our results need to be confirmed in other populations and with direct measurements of hormone status, but are encouraging for further research on the effect of sex hormones on the development of atopy.

The study was supported by the Finnish Academy, National Public Health Institute and University of Oulu.

1 Björksten B. The environmental influence on childhood asthma. Allergy 1999;54:17-23.

2 Wjst M, Dold S. Is asthma an endocrine disease? Pediatr Allergy Immunol 1997;8:200-4.

3 Apter D, Vihko R. Premenarcheal endocrine changes in relation to age at menarche. Clin Endocrinol 1985;22:75360

4 Apter D, Reinila M, Vihko R. Some endocrine characteristics of early menarche, a risk factor for breast cancer, are preserved into adulthood. Int 7 Cancer 1989;44:783-7.

5 Rantakallio P. Groups at risk in low birth weight children and perinatal mortality. Acta Pediatric Scand 1969;Suppl

6 Xu B, Järvelin M-R, Pekkanen J. Body build and atopy. $\mathcal{F}$ Allergy Clin Immunol 2000;105:393-4

7 Cocchiara R, Albeggiani G, Di Trapani G, et al. Modulation of rat peritoneal mast cell and human basophil histamine release by estrogens. Int Arch Allergy Appl Immunol 1990;93:192-7.

8 Kalogeromitros D, Katsarou A, Armenaka M, et al. Influence of the menstrual cycle on skin-prick test reactions to histamine, morphine and allergen. Clin Exp Allergy 1995;25:461-6.

9 Grossman JC Regulation of the immune system by sex steroids. Endocine Rev 1984;5:435-55.

10 Petridou E, Syrigou E, Toupadaki N, et al. Determinants of age at menarche as early predictors of breast cancer risk. Int fCancer 1997;68:193-8.
Department of

Infectious Diseases

and Clinical

Epidemiology, Monash

Medical Centre,

Clayton 3168, Victoria,

Australia

R L Stuart

N Bennett

M L Grayson

Department of

Epidemiology and

Preventive Medicine,

Monash Medical

School, Alfred

Hospital, Prahan,

Victoria, Australia

M L Grayson

R L Stuar

A Forbes

Correspondence to: Dr R Stuart

email: rhonda.stuart@

med.monash.edu.au

Received 18 October 1999

Returned to authors

7 January 2000

Revised version received

13 March 2000

Accepted for publication

26 April 2000

\title{
A paired comparison of tuberculin skin test results in health care workers using $5 \mathrm{TU}$ and $10 \mathrm{TU}$ tuberculin
}

\author{
Rhonda L Stuart, Noleen Bennett, Andrew Forbes, M Lindsay Grayson
}

\begin{abstract}
Background-Historically, 10 TU has been employed in Australia and the United Kingdom to perform the tuberculin skin test (TST). However, this makes it difficult to compare the rates of TST positivity with other countries such as the USA who use $5 \mathrm{TU}$. To assess the impact of the dose of tuberculin on the TST a comparison was made of TST responses in health care workers given a TST with both 5 and 10 TU.
\end{abstract}

Methods-Two TSTs were performed simultaneously in each health care worker using 5 and 10 TU. Each dose was randomly assigned in a blinded manner to the right or left forearm and read at 48-72 hours by a single nurse who was blinded to the assignment of the 5 and 10 TU doses. Results-A total of 128 health care workers were enrolled, 119 (93\%) of whom had a past history of BCG vaccination. The overall mean difference in paired reaction sizes for the two doses was $1.5 \mathrm{~mm}$ with $95 \%$ limits of agreement of -3.6 to $6.5 \mathrm{~mm}$. Conclusion-A slightly larger TST reading was seen with 10 TU than with 5 TU. The mean difference of $1.5 \mathrm{~mm}$ between the two doses should be considered when comparing rates of TST positivity between countries who use different doses of tuberculin to perform the tuberculin skin test.

(Thorax 2000;55:693-695)

Keywords: tuberculin skin test; health care workers; dosage

The tuberculin skin test (TST) is the most commonly used test to detect previous $\mathrm{Myco}$ bacterium tuberculosis infection, being used in epidemiological surveys, clinical evaluation of 
Table 1 Comparison of TST measurements using 5 and 10 TU tuberculin

\begin{tabular}{llccll}
\hline \multirow{2}{*}{$10 \mathrm{TU}$ dose } & \multicolumn{5}{l}{$5 \mathrm{TU}$ dose } \\
\cline { 2 - 5 } & $0 \mathrm{~mm}$ & $1-9 \mathrm{~mm}$ & $10-14 \mathrm{~mm}$ & $15-19 \mathrm{~mm}$ & $\geqslant 20 \mathrm{~mm}$ \\
\hline $0 \mathrm{~mm}$ & $31(24)$ & $1(1)$ & 0 & 0 & 0 \\
$1-9 \mathrm{~mm}$ & $11(9)$ & $42(33)$ & $3(2)$ & 0 & 0 \\
$10-14 \mathrm{~mm}$ & 0 & $17(13)$ & $14(11)$ & $1(0.8)$ & 0 \\
$15-19 \mathrm{~mm}$ & 0 & 0 & $2(1.5)$ & $3(2)$ & 0 \\
$\geqslant 20 \mathrm{~mm}$ & 0 & 0 & $1(0.8)$ & $2(1.5)$ & 0 \\
\hline
\end{tabular}

Values are no. (\%).

patients with suspected active tuberculosis, and assessment for preventive antituberculous drug therapy. Unfortunately the TST has many variables that may affect its interpretation and result. These include variation in tuberculin dose and formulation, experience and technique in application, the effect of previous BCG vaccination, subject age, recent vaccination with live vaccines, and underlying immunosuppression. ${ }^{1}$

In the 1940s and 1950s the "optimal" dosage of tuberculin was determined. Persons with and without a history of exposure to tuberculosis were tested with increasing doses of tuberculin and the cumulative proportion of reactors was calculated. From these studies it was concluded that $5 \mathrm{TU}$ was the dose that gave the "best" balance between sensitivity and specificity. $^{2}$

Historically, $10 \mathrm{TU}$ has been employed in Australia and the United Kingdom to perform the TST, ${ }^{34}$ while in the United States $5 \mathrm{TU}$ is the standard dose used. ${ }^{5}$ Because of these dosage differences in performing the TST, comparison of TST results between countries may be difficult. In particular, comparisons of large multicentre TST studies of health care workers in different countries have been complicated by these differences in TST dosage. ${ }^{67}$ To assess the impact of the tuberculin dose on the TST we compared the TST responses in a cohort of BCG vaccinated and unvaccinated health care workers who received a TST with both 5 and 10 TU simultaneously.

\section{Methods}

Health care workers within the Southern Healthcare Network were informed of the study via table drops and individual mailings. Those who had previous TST readings of 5-19 $\mathrm{mm}$ were particularly encouraged to enrol. This was aimed at increasing the likelihood of positive results to both the 5 and $10 \mathrm{TU}$ doses. The upper limit of $19 \mathrm{~mm}$ was chosen to decrease the risk of large reactions occurring in individuals who had previously tested positive. Health care workers completed a questionnaire at the time of TST placement documenting age, occupation, and prior BCG status and timing.

TSTs were given by one of two trained personnel. Two TSTs were performed simultaneously in each health care worker using a 5 and $10 \mathrm{TU}$ dose. Each dose was assigned to the right or left forearm based on whether their home address was an odd or even number. To minimise reader variability all results were read by a single nurse who was blinded to the assignment of the 5 and $10 \mathrm{TU}$ doses. The transverse diameter of induration at 4872 hours was measured by the palpation method.

The study was approved by the ethics committee of the Southern Healthcare Network.

The results of the 10 TU test were used to assess whether the individual needed further investigation and follow up. According to the Australian and British guidelines, a TST was classified as strongly positive if induration of $\geqslant 15 \mathrm{~mm}$ was detected in a person who had previously received $B C G$ and $\geqslant 10 \mathrm{~mm}$ in those without such a history. ${ }^{4}$ These individuals underwent chest radiography and were offered individual consultation with a specialist physician.

Data were analysed using the two tailed paired samples $t$ test. A residual-like plot of the difference between the measurements against their mean as described by Bland and Altman ${ }^{9}$ was also employed since this method allows the detection of differences between the two doses at different diameters of induration.

\section{Results}

One hundred and twenty eight health care workers were enrolled (102 women) of overall median age 42 years (range 21-65), 119 (93\%) of whom had a past history of BCG vaccination (mean number of years since $\mathrm{BCG}=26$; range $2-47)$. There were 70 nurses (55\%), seven physicians (6\%), 12 laboratory workers (9\%), and 40 allied health workers $(30 \%)$. Thirty one had readings for both the 5 and $10 \mathrm{TU}$ doses recorded as zero; the remaining 97 had a least one recordable response for either the 5 or 10 TU dose.

Eight BCG vaccinated health care workers had TSTs of $\geqslant 15 \mathrm{~mm}$ with $10 \mathrm{TU}$ and $\geqslant 10 \mathrm{~mm}$ with the $5 \mathrm{TU}$ dose. An additional subject without a history of BCG vaccination had TSTs of $10 \mathrm{~mm}$ and $11 \mathrm{~mm}$ with the 10 TU and 5 TU doses, respectively. All nine individuals had normal chest radiographs and no evidence of active tuberculosis.

Using the USA guidelines ${ }^{10}$ to determine TST positivity ( $\geqslant 10 \mathrm{~mm}$ regardless of BCG status), 40 (31\%) subjects would have been considered positive with 10 TU and $26(20 \%)$ positive with $5 \mathrm{TU}$. In comparison, using the Australian and UK guidelines ${ }^{4}{ }^{8}(\geqslant 10 \mathrm{~mm}$ if no history of BCG vaccination; $\geqslant 15 \mathrm{~mm}$ if previously vaccinated), eight $(0.06 \%)$ and seven $(0.05 \%)$ would have been positive with the 10 TU and 5 TU doses, respectively.

A comparison of the diameter of induration obtained from the two doses is shown in table 1. Most of the health care workers $(66 \%)$ had a TST of $<10 \mathrm{~mm}$. Generally, the $10 \mathrm{TU}$ dose gave a larger TST reading than the 5 TU dose, but in 16 cases $(13 \%)$ the $5 \mathrm{TU}$ reading was greater. The overall mean difference in paired reaction sizes for the two doses was $1.5 \mathrm{~mm}$ (95\% CI 1.0 to $1.9 ; \mathrm{p}<0.001$ ). In the 97 health care workers who had a least one recordable TST response to the 5 or $10 \mathrm{TU}$ doses, the mean difference in reaction was $1.9 \mathrm{~mm}(95 \%$ CI 1.4 to $2.5 ; \mathrm{p}<0.001$ ). 


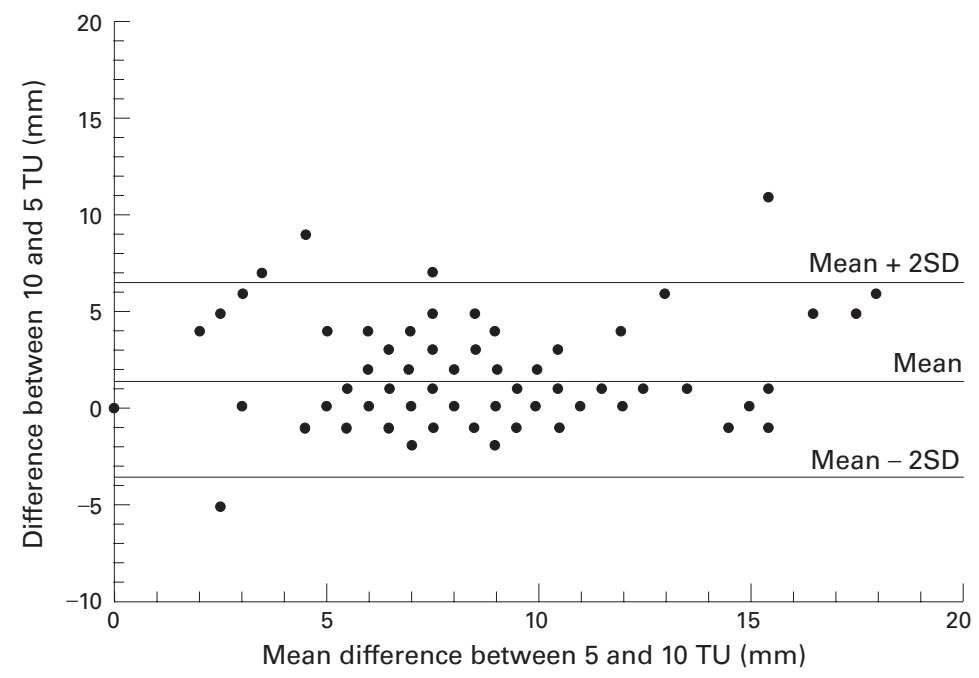

Figure 1 Plot of the difference between 5 and 10 TU doses versus mean

Differences in results between the two doses plotted against the mean of each subject's result are shown in fig 1 . Using the Bland-Altman method $^{9}$ for assessing agreement between the two doses, the $95 \%$ limits of agreement were -3.6 to $6.5 \mathrm{~mm}$, meaning that $95 \%$ of paired results are expected to have differences within this range. Notably, there was no relationship between this difference in TST response and the mean diameter of induration, suggesting that the difference in 5 and 10 TU results did not change significantly with increasing TST response. There was, however, an association between the mean difference in the results and the age of the health care worker $(r=0.21, \mathrm{p}=0.01)$, but not with years since last BCG vaccination $(r=0.1, \mathrm{p}$ $=0.29$; data not shown).

\section{Discussion}

The tuberculin skin test has long been an important aid in the diagnosis of tuberculous infection and disease. However, different countries use different doses of PPD for screening so that there is no uniformity. ${ }^{3-5}$ Only one previous study has compared the differences seen when 5 or 10 TU of PPD is employed in the TST. $^{11}$ However, this 1954 World Health Organisation study assessed a Danish cohort with no history of previous BCG vaccination who received either a 5 or 10 TU TST, but not both doses simultaneously. The average $10 \mathrm{TU}$ TST result was $2-3 \mathrm{~mm}$ larger than the $5 \mathrm{TU}$ dose, but the unpaired nature of this study design limited the conclusions that could be drawn from these data.

Our results show that there is a statistically significant but small mean difference of
$1.5 \mathrm{~mm}$ obtained when the 5 and $10 \mathrm{TU}$ doses are compared within individuals. This small increase in TST result obtained with the use of the $10 \mathrm{TU}$ dose was large enough to increase the number of positive individuals by $11 \%$ (14/ 128) using a $10 \mathrm{~mm}$ cut off. If, however, a $15 \mathrm{~mm}$ cut off was used, as is recommended in Australia and the UK, only one extra positive reading was seen with $10 \mathrm{TU}$ compared with the 5 TU dose. Given that most of our health care workers had a past history of BCG vaccination, it would be of interest to assess the differences in a group of non-vaccinated individuals. The lack of a gold standard to determine who is truly infected with tuberculosis when interpreting a TST result remains an issue for the significance of our study, as with other authors on this topic.

These data suggest that, in subjects having a TST, the impact of using 5 or $10 \mathrm{TU}$ to perform the test when comparing studies using one or the other of these doses is likely to be limited. Nevertheless, these differences should be borne in mind and our data suggest that studies using $10 \mathrm{TU}$ should be calibrated back by $1.5 \mathrm{~mm}$ to enable comparison with studies employing $5 \mathrm{TU}$. Whether the previously perceived advantages of using 10 TU in countries such as Australia and the UK really justify the potential for confusion when interpreting the result remains uncertain.

1 Huebner RE, Schein MF, Bass JB. The tuberculin skin test. Clin Infect Dis 1993;17:968-75.

2 Rieder HL. Epidemiologic basis of tuberculosis control. IUATLD 1999:30-34.

3 National Health and Medical Research Council. The Australian Immunisation Handbook. 6th edn. Canberra: Australian Government Publishing Service, 1997.

4 British Thoracic Society. Guidelines on the management of tuberculosis and HIV infection in the United Kingdom. BMF 1992;304:1231-3.

5 American Thoracic Society. Diagnostic standards and classification of tuberculosis. Am Rev Respir Dis 1990;142: 725-35.

6 Bailey TC, Fraser VJ, Spitnagel EL, et al. Risk factors for a positive tuberculin skin test among employees of an urban, midwestern teaching hospital. Ann Intern Med 1995;122: $580-5$.

7 Horowitz HW, Luciano BB, Kadel JR, et al. Tuberculin skin test conversion in hospital employees vaccinated with bacille Calmette-Guerin: recent Mycobacterium tuberculosis infection or booster effect? Am F Infect Control 1995;23: $181-7$.

8 National Health and Medical Research Council. Tuberculosis in Australia and New Zealand into the 1990s. Canberra: Australian Government Publishing Service, 1990.

9 Bland J, Altman D. Statistical methods for assessing agreement between two methods of clinical measurement. Lancet 1986;i:307-10.

10 Centers for Disease Control. The use of preventative therapy for tuberculosis infection in the United States. MMWR 1990;39(RR-8):9-12.

11 World Health Organisation. The 5TU versus the $10 \mathrm{TU}$ intradermal tuberculin test. Bulletin WHO (BCG vaccination). Geneva: WHO, 1955: 169-71. 